\title{
Particles and Shadows: A Generalized Path-Integral Approach to Nonrelativistic Field Theories
}

\author{
R. Rosenfelder
}

Paul Scherrer Institute, CH-5232 Villigen PSI, Switzerland.

\begin{abstract}
Luttinger and Lu have derived a variational method for representing the Yukawa interaction of bosons and fermions in nonrelativistic field theories by a general potential between fermions and fictitious particles ("shadows"). In the present work this is considerably improved by applying Jensen's inequality only once and representing the kinetic term in the path-integral average as a quantum-mechanical $N$-body problem using the replica technique. Various weak- and strongcoupling approximations as well as variational bounds for this term are discussed.
\end{abstract}




\section{Introduction}

Many non-relativistic systems in solid state and nuclear physics can be described by field-theoretical models of fermions interacting with bosons. The Hamiltonian of such a system of $A$ fermions with mass $m$ usually has the form

$$
\hat{H}=\sum_{i=1}^{A} \frac{\mathbf{p}_{i}^{2}}{2 m}+\int d^{3} k \omega(\mathbf{k}) a^{\dagger}(\mathbf{k}) a(\mathbf{k})+\sqrt{\alpha} \sum_{i=1}^{A} \int \frac{d^{3} k}{(2 \pi)^{3}}\left[f(\mathbf{k}) a^{\dagger}(\mathbf{k}) e^{-i \mathbf{k} \cdot \mathbf{x}_{i}}+\text { h.c }\right]
$$

when the interaction is assumed to be spin-independent. Here $a(\mathbf{k})$ and $a^{\dagger}(\mathbf{k})$ are annihilation and creation operators for the bosons (phonons or mesons), $\omega(\mathbf{k})$ denotes the boson frequency and $\alpha$ is a dimensionless coupling constant between fermions and bosons. For the well-known polaron problem $[1-4]$ the function $f(\mathbf{k})$ is given by

$$
f(\mathbf{k})=i\left(\frac{4 \pi}{\sqrt{2}}\right)^{1 / 2} \frac{1}{|\mathbf{k}|}
$$

for the meson-nucleon problem see refs. $[5,6]$. In the following I will only consider the zero-momentum self energy of a single fermion $(A=1)$ interacting with any number of bosons.

Feynman [7] observed that the bosons can be eliminated analytically since they enter at most quadratically in the Hamiltonian (1). In the path-integral formulation one obtains an effective action for the fermion which for large euclidean time $\beta$ is given by

$$
S=\int_{0}^{\beta} d t \frac{m}{2} \dot{\mathbf{x}}^{2}-\alpha \int_{0}^{\beta} d t \int_{0}^{\beta} d t^{\prime} \int \frac{d^{3} k}{(2 \pi)^{3}}|f(\mathbf{k})|^{2} e^{-\omega(\mathbf{k})\left|t-t^{\prime}\right|} e^{i \mathbf{k} \cdot\left(\mathbf{x}(t)-\mathbf{x}\left(t^{\prime}\right)\right)} \equiv S_{0}+S_{1} .
$$

With the elimination of the bosonic degrees of freedom one is left with a one-body effective theory which, however, is highly non-local in time due to the retardation effects. This prevents a Hamiltonian formulation and any further exact analytical treatment. Instead Feynman used a variational formulation for the partition function

$$
Z=\int_{\mathbf{x}(0)=\mathbf{x}(\beta)} \mathcal{D}[\mathbf{x}] e^{-S[\mathbf{x}]}
$$

based on Jensen's inequality $\langle\exp (-S)\rangle \geq \exp (-<S>)$. This yields an upper limit for the ground state energy (self-energy at zero momentum)

$$
E_{0} \leq E_{\text {trial }}+\lim _{\beta \rightarrow \infty} \frac{1}{\beta}<S-S_{\text {trial }}>S_{\text {trial }}
$$

where $E_{\text {trial }}$ is the energy corresponding to some suitably chosen trial action $S_{\text {trial }}$ and the average is performed with respect to $S_{\text {trial }}$.

The trial action proposed by Feynman for the polaron problem is that of a retarded harmonic oscillator and in the usual polaron units $(m=\omega=1)$ it is given by

$$
S_{F}=\int_{0}^{\beta} d t \frac{1}{2} \dot{\mathbf{x}}^{2}+\frac{w}{4}\left(v^{2}-w^{2}\right) \int_{0}^{\beta} d t \int_{0}^{t} d t^{\prime} e^{-w\left(t-t^{\prime}\right)}\left|\mathbf{x}(t)-\mathbf{x}\left(t^{\prime}\right)\right|^{2}
$$


where $v$ and $w$ are variational parameters. They are determined by minimizing eq. (5) which becomes

$$
E_{0} \leq E_{F}=\frac{3}{4 v}(v-w)^{2}-\frac{v \alpha}{\sqrt{\pi}} \int_{0}^{\infty} d \tau \frac{e^{-\tau}}{\left[w^{2} \tau+\left(v^{2}-w^{2}\right)\left(1-e^{-v \tau}\right) / v\right]^{1 / 2}} .
$$

Feynman's approach is the best analytical approximation which works for both weak- and strongcoupling with a maximal error for the ground-state energy of only $2.2 \%$ for large $\alpha$.

\section{Luttinger and Lu's Approach and Improvement}

It was one of the motivations $[7,8]$ for the ansatz (6) that it results from "integrating out" a fictitious particle with mass

$$
M=\frac{v^{2}-w^{2}}{w^{2}}
$$

and coordinate $\mathbf{R}$ which couples harmonically to the electron

$$
V(\mathbf{R}-\mathbf{x})=\frac{1}{2} M w^{2}(\mathbf{R}-\mathbf{x})^{2}
$$

This "shadow" particle simulates the cloud of phonons around the electron in the crystal.

Luttinger and Lu ( LL ) [9] have generalized this idea to an arbitrary potential $V(\mathbf{R}-\mathbf{x})$ and have shown that for large coupling constants the best effective potential is not harmonic (like in Feynman's approximation) but Coulomb-like. In this way they obtained the exact strong-coupling limit for the ground-state energy due to Pekar [10] and Miyake [11]. Their method is based on multiplying eq. (4) by

$$
1=\frac{\int \mathcal{D}[\mathbf{R}] \exp \left(-T[\mathbf{R}-\mathbf{x}]+T_{1}[\mathbf{R}-\mathbf{x}]\right)}{\int \mathcal{D}[\mathbf{R}] \exp \left(-T_{0}[\mathbf{R}]\right)}
$$

where

$$
T[\mathbf{R}-\mathbf{x}]=\int_{0}^{\beta} d t\left(\frac{M}{2} \dot{\mathbf{R}}^{2}+V(\mathbf{R}-\mathbf{x})\right) \equiv T_{0}[\mathbf{R}]+T_{1}[\mathbf{R}-\mathbf{x}]
$$

is the action of a shadow particle coupled to the fermion. They then invoked again Jensen's inequality for the combined path integral and determined the optimal potential function $V(\mathbf{r})$ and mass parameter $M$. An important observation made by Luttinger and $\mathrm{Lu}$ is that the non-gaussian path integrals which necessarily occur in their approach can be evaluated by solving the corresponding quantum-mechanical problem (i.e. the Schrödinger equation). For example

$$
\int \mathcal{D}[\mathbf{x}] \mathcal{D}[\mathbf{R}] \exp \left(-S_{0}[\mathbf{x}]-T[\mathbf{R}-\mathbf{x}]\right)=\text { const. } \times \operatorname{Tr}\left(e^{-\beta \hat{H}_{2}}\right)
$$

where

$$
\hat{H}_{2}=\frac{\mathbf{P}^{2}}{2(M+m)}+\frac{\mathbf{p}^{2}}{2 \mu}+V(\mathbf{r})
$$

is the two-body Hamiltonian in center-of-mass and relative coordinates with reduced mass $\mu=$ $m M /(M+m)$.

However, applying the inequality twice, i.e. to particle and shadow leads to a rather bad approximation except for large coupling constants. This is best seen for a harmonic interaction where 
Luttinger and Lu obtain nearly the same result (7) as Feynman but with the first term (which I will call "kinetic" part) being replaced by $3 v / 4$. This leads to much more repulsion at small $\alpha:$ instead of Feynman's $(v-w)^{2} / v=\mathcal{O}\left(\alpha^{2}\right)$ Luttinger and Lu only have $v=\mathcal{O}(1)$. The difference does not matter at large coupling constants when $v>>w$ but is essential at low and intermediate coupling. In consequence $E_{L L}$ (in the so-called ground-state approximation) is only better than $E_{F}$ for $\alpha>34$ despite the fact that an arbitrary potential $V(\mathbf{r})$ has been allowed for the variational principle. This renders the LL approach basically a complicated strong coupling expansion.

To improve Luttinger and Lu's treatment and to exploit the full flexibility of a general potential it seems necessary to use Jensen's inequality only once. This can be accomplished by defining the trial action directly as a result of the elimination of a shadow particle:

$$
\exp \left(-S_{\text {trial }}[\mathbf{x}]\right)=\int \mathcal{D}[\mathbf{R}] e^{-S_{0}[\mathbf{x}]-T[\mathbf{R}-\mathbf{x}]}
$$

Note that this is translationally invariant and reduces to Feynman's ansatz (6) for a harmonic interaction. One then has to evaluate the individual averages in eq. (5).

The simplest term is the trial energy $E_{\text {trial }}$ : using eq. (11a) one immediately obtains

$$
E_{\text {trial }}=\epsilon_{0}^{(2)}
$$

where $\epsilon_{0}^{(2)}$ (I suppress the superscript in most of the following) is the lowest eigenvalue of the twobody Hamiltonian (12). Note that after separation of the center-of-mass motion this reduces to the determination of the lowest energy of a particle of mass $\mu$ in an (up to now) arbitrary potential $V(r)$ - a problem which can be solved easily by standard numerical methods.

The evaluation of $\left\langle S_{1}\right\rangle_{S_{\text {triel }}}$ proceeds similarly using the equivalence

$$
\int \mathcal{D}[\mathbf{x}] \mathcal{D}[\mathbf{R}] \mathcal{O}_{1}\left(t_{1}\right) \mathcal{O}_{2}\left(t_{2}\right) \exp \left(-S_{0}[\mathbf{x}]-T[\mathbf{R}-\mathbf{x}]\right)=\text { const. } \times \operatorname{Tr}\left(\mathcal{T} \hat{\mathcal{O}}_{1 H}\left(t_{1}\right) \hat{\mathcal{O}}_{2 H}\left(t_{2}\right) e^{-\beta \hat{H}_{2}}\right)
$$

where $T$ is the time-ordering symbol and $\hat{\mathcal{O}}_{H}(t)$ denotes the corresponding Heisenberg operator. A straight-forward evaluation yields the same expression as given by $\mathrm{LL}$

$$
<S_{1}>_{S_{\text {trisl }}}=-\alpha \int \frac{d^{3} k}{(2 \pi)^{3}}|f(\mathbf{k})|^{2} \sum_{n} \frac{|<0| \exp (i \mu \mathbf{k} \cdot \mathbf{r} / m)|n>|^{2}}{\omega(\mathbf{k})+\epsilon_{n}-\epsilon_{0}+\mathbf{k}^{2} / 2(M+m)} .
$$

Note that here the knowledge of the full spectrum of $\hat{H}_{2}$ is needed which makes the numerical evaluation of eq. (15) for a general potential a major task. However, as observed by Luttinger and Lu each term in the spectral sum gives a positive contribution so that one still has an upper bound on the energy if only a finite number of states are retained. In the strong-coupling limit already the ground state contribution leads to the exact Pekar result.

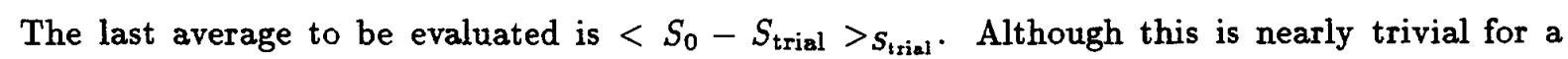
harmonic interaction, i.e. in Feynman's approximation ${ }^{1}$, it turns out to be the most challenging problem for a general potential and it is the major topic of this work. This is due to the fact that the trial action $S_{\text {trial }}$ appears for the first time directly and not only in the form $\exp \left(-S_{\text {trial }}\right)$ where

\footnotetext{
${ }^{1}$ In particular, if the Fourier path integral form is used [12].
} 
it can readily be transformed into quantum-mechanical expectation values as in eqs. (11). Using the definition (13) for the trial action an alternative formulation of the problem is to evaluate the non-standard path integral

$$
\begin{aligned}
<S_{0}-S_{\text {trial }}>_{S_{\text {trial }}}= & \int \mathcal{D}[\mathbf{x}] \mathcal{D}[\mathbf{R}] \exp \left(-S_{0}[\mathbf{x}]-T[\mathbf{R}-\mathbf{x}]\right) \log \left[\int \mathcal{D}\left[\mathbf{R}_{1}\right] \exp \left(-T\left[\mathbf{R}_{1}-\mathbf{x}\right]\right)\right] \\
& \cdot\left(\int \mathcal{D}[\mathbf{x}] \mathcal{D}[\mathbf{R}] \exp \left(-S_{0}[\mathbf{x}]-T[\mathbf{R}-\mathbf{x}]\right)\right)^{-1}
\end{aligned}
$$

\section{Replicas}

Obviously it is the logarithm in eq. (16) which prevents a formulation in terms of stationary quantum mechanics as in the previous terms. A similar problem arises in the linked cluster theorem which states that $\log Z$ is given by the sum of connected diagrams. An elegant method to prove this theorem is the replica technique [13] based on the representation

$$
\log Z=\lim _{N \rightarrow 0} \frac{\partial}{\partial N} Z^{N} .
$$

For integer $N, Z^{N}$ can again be represented as quantum-mechanical expectation value with a Hamiltonian containing $N$ "replicas" of the original particles. Applying this trick to the evaluation of $<S_{0}-S_{\text {trial }}>$ one obtains after renumbering $N \rightarrow N+1$

$$
<S_{0}-S_{\text {trial }}>_{S_{\text {trial }}}=\left.\frac{\partial}{\partial N} \operatorname{Tr}\left(e^{-\beta \hat{H}_{N+1}}\right)\right|_{N=1}
$$

where the $(N+1)$-body Hamiltonian is given by

$$
\hat{H}_{N+1}=\frac{\mathbf{p}^{2}}{2 m}+\sum_{i=1}^{N}\left(\frac{\mathbf{p}_{i}^{2}}{2 M}+V\left(\mathbf{x}-\mathbf{R}_{i}\right)\right) .
$$

This is similar to an atom consisting of $\mathrm{N}$ electrons which have no interaction among themselves but only with the nucleus (which in our case is the single fermion). Transforming to the center-of-mass (which is not the "nucleus") and to coordinates relative to the "nucleus" one obtains $[14,15]$

$$
\hat{H}_{N+1}=\frac{\mathbf{P}^{2}}{2(N M+m)}+\sum_{i=1}^{N}\left(\frac{\mathbf{p}_{i}^{2}}{2 \mu}+V\left(\mathbf{r}_{i}\right)\right)+\sum_{i \neq j} \frac{\mathbf{p}_{i} \cdot \mathbf{p}_{j}}{2 m} .
$$

The last term - the so-called Eckart term - is a genuine two-body interaction and responsible for the coupling among the particles. Otherwise the ground-state energy $E_{N}$ would be just the sum of single-particle energies $\epsilon_{0}$ and the quantity

$$
\Omega \equiv E_{\text {trial }}+\lim _{\beta \rightarrow \infty} \frac{1}{\beta}<S_{0}-S_{\text {trial }}>S_{\text {trial }}=\epsilon_{0}-\left.\frac{\partial}{\partial N} E_{N}\right|_{N=1}=-\left.\frac{\partial}{\partial N} \frac{E_{N}}{N}\right|_{N=1}
$$

would vanish identically. In the following $\Omega$ will be called "kinetic" term as it is the part of the variational bound for the ground-state energy

$$
E_{0} \leq \Omega+<S_{1}>_{S_{\text {trial }}}
$$


which does not depend explicitly on the coupling constant $\alpha$. It is precisely the insufficient bound for $\Omega$ which makes the more general approach of Luttinger and Lu inferior compared to Feynman's. By using the replica trick the problem has now been transformed into determining the chemical potential $\mu=\partial E_{N} / \partial N$ at $N=1$ with the Hamiltonian (20). Equivalently from eq. (21) one has to determine the energy per particle beyond the Hartree approximation, i.e. the correlation energy of the system. Of course, for the harmonic interaction (8) this can be done exactly by finding the normal modes [18]. The result is

$$
E_{N}^{\text {harmonic }}=\frac{3}{2} w(N-1)+\frac{3}{2} w \sqrt{N M+m} .
$$

Using eq. (8a) it is easily checked that this leads to the correct Feynman result

$$
\Omega^{\text {harmonic }}=\frac{3}{4 v}(v-w)^{2} .
$$

For a general potential and for small coupling constants the kinetic term $\Omega$ can be calculated systematically considering the Eckart term as perturbation. This is possible because the "nucleus" practically does not move in this limit $(m>>M)$. The simplest way of dealing with $N$ bosons (which can condense) is to treat them as fermions and to assign an internal "spin" to them with $N$ magnetic components [13]. Each loop in a Goldstone diagram for the ground state energy then carries a factor $N$. The particular separable form of the Eckart term as two-body interaction leads to considerable simplifications: only an even number of interactions on any particle or hole line is possible due to parity conservation. In addition, one-loop diagrams can be dismissed for the perturbative evaluation of $\Omega$ since linear terms in $N$ do not contribute to eq. (21).

It was already noted that the Hartree term gives zero contribution to $\Omega$. There are no first-order Goldstone diagrams. In second order the only diagram which survives is the one shown in Fig. 1 and its contribution is given by

$$
\Omega^{(2)}=\frac{1}{2 m^{2}} \sum_{a, b} \frac{\left|\mathbf{p}_{0 a} \cdot \mathbf{p}_{0 b}\right|^{2}}{\epsilon_{a}+\epsilon_{b}-2 \epsilon_{0}}
$$

where $\mathbf{p}_{0 a}=\langle 0|\mathbf{p}| a>$ is the single-particle matrix element of the momentum operator in the basis of eigenstates of $\hat{H}_{2}$. In third order one finds that from the 14 Goldstone diagrams for the energy [17] only the four shown in Fig. $2 \mathrm{a}-2 \mathrm{~d}$ contribute with relative weight $-2,1,1$ and 1 , respectively. This gives

$$
\Omega^{(3)}=\frac{1}{m^{3}} \sum_{a, b, c} \frac{\mathbf{p}_{0 b} \cdot \mathbf{p}_{0 c} \mathbf{p}_{c 0} \cdot \mathbf{p}_{0 a} \mathbf{p}_{a 0} \cdot \mathbf{p}_{c 0}}{\left(\epsilon_{b}+\epsilon_{c}-2 \epsilon_{0}\right)\left(\epsilon_{a}+\epsilon_{b}-2 \epsilon_{0}\right)}
$$

As a check it is useful to evaluate eqs. $(25,26)$ for the harmonic interaction (8). This is very easy because only one excited $\mathrm{p}$-state contributes to each sum. One finds $\Omega^{(2)}=3\left(v^{2}-w^{2}\right)^{2} / 16 v^{3}$ and $\Omega^{(2)}+\Omega^{(3)}=\Omega^{(2)}\left(1+\left(v^{2}-w^{2}\right) / 2 v^{2}\right)$ which agrees with the exact result up to terms of $\mathcal{O}\left(\alpha^{3}\right)$ and $\mathcal{O}\left(\alpha^{4}\right)$, respectively. The ring diagrams shown in Figs. 1 and 2 can be summed by diagonalizing the Hamiltonian (20) in a basis made up by the zero'th and first-order perturbative wavefunction. As shown in Appendix A one obtains

$$
\Omega^{\text {ring }}=\frac{\Omega^{(2)}}{1-\Omega^{(3)} / \Omega^{(2)}}
$$


which is somewhat better than the perturbative result for large couplings constants: for a harmonic interaction it gives one half of the exact result compared to $3 / 8$ of the exact result when $\Omega^{(2)}$ and $\Omega^{(2)}$ are added.

Still the resummation in eq. (27) is insufficient for large coupling constants since it only contains particle-hole interctions and leaves out particle-particle interactions. In this limit the replicas become very massive and do not move much whereas the fermion is now the light particle. This is a situation reminiscent of the treatment of nuclear motion in molecules when the Born-Oppenheimer approximation applies. As is well known [19] the energy of the electrons (the fermion in our case) depends parametrically on the coordinates of the nuclei (the replicas) and serves as a potential for the latter ones. For $M \rightarrow \infty$ the ground state energy is then just the minimum of $\epsilon_{0}\left(\mathbf{R}_{1}, \mathbf{R}_{2} \ldots \mathbf{R}_{N}\right)$ with corrections coming from harmonic vibrations around the minimal points. As the replicas do not interact with each other, these minimal positions are all the same. Taking into account that the difference between $m$ and $\mu$ is negligible for large $M$ it then follows from eq. (19) that the ground state energy of the system is $\epsilon_{0}^{(2)}[V \rightarrow N V]$. Using the Hellmann-Feynman theorem [20,14] to perform the $N$-differentiation we therefore obtain from eq. (21)

$$
\Omega \stackrel{M>>m}{\longrightarrow} \epsilon_{0}-<0|V| 0>=<0\left|\frac{\mathrm{p}^{2}}{2 \mu}\right| 0>,
$$

i.e. the kinetic term is given by the mean kinetic energy of the particle in the ground state of $\hat{H}_{2}$. As shown by Luttinger and Lu this leads to the correct Pekar limit for the self-energy at large coupling. However, in the LL approach eq. (28) is used for all coupling constants which is not justified as the rôle of heavy and light particles reverses at smaller coupling. This is illustrated in Fig. 3 .

\section{Variational Bounds}

Calculating the kinetic term $\Omega$ for weak and strong coupling is not very useful for several reasons. First, perturbative calculations can be easily performed for the full field-theoretical problem defined by eq. (1) so that a perturbative calculation in an approximate context does not make much sense. In the polaron problem the same applies for the strong coupling case. It is exactly the intermediate coupling case where discrepancies exist between Monte-Carlo calculations [12] and approximate analytical methods [21]. Second, by evaluating $\Omega$ approximately the variational bound for the self-energy is lost in most cases.

An exception is the resummation (27) of ring diagrams which can be shown to yield a lower bound for $\Omega$ (see Appendix A). However, what really is needed for the variational principle (22) is an upper bound. This can be obtained in several ways. One is by recognizing that $\Omega=\epsilon_{0}-\mu$ is the thermodynamical potential at zero temperature and mean particle number $N=1$ for which well-known variational bounds exist $[22,23]$. I have not succeeded in finding simple ansätze which lead to useful results. Another possibility is to derive a lower bound for

$$
\left.\frac{\partial}{\partial N} E_{N}\right|_{N=1}=\lim _{N \rightarrow 1} \frac{E(N)-E(1)}{N-1}
$$


Since $E(1)=\epsilon_{0}$ is known exactly this requires a lower bound for the ground state energy such as Temple's bound $[24,14]$

$$
E_{0} \geq \bar{E}-\frac{\left\langle\psi\left|\left(\hat{H}_{N+1}-\bar{E}\right)^{2}\right| \psi>\right.}{E_{1}-\bar{E}} .
$$

Here $\bar{E}=\left\langle\psi\left|\hat{H}_{N+1}\right| \psi\right\rangle$ and $E_{1}$ denotes the exact first excited state energy of the $(N+1)$ - particle system. Since the latter one is not known one needs also a lower bound for it. A very crude one is obtained [25] by observing that the Hamiltonian of the system can be written as

$$
H_{N+1}=\sum_{i=1}^{N}\left[\frac{\mathbf{p}_{i}^{2}}{2 M}+V\left(\mathbf{r}_{i}\right)\right]+\frac{1}{2 m}\left(\sum_{i=1}^{N} \mathbf{p}_{i}\right)^{2} \geq \sum_{i=1}^{N} \tilde{h}_{i}
$$

so that all energies are bounded from below by $N \tilde{\epsilon}_{n}$ where $\tilde{\epsilon}_{n}$ are the eigenvalues of the one-body Hamiltonian $\tilde{h}=\mathbf{p}^{2} / 2 M+V(\mathbf{r})$. Using eqs. (21) and (29) one then obtains an upper bound for $\Omega$ provided the denominator in eq. (30) stays positive. For example, taking the Hartree wave functions as trial states leads to

$$
\Omega \leq \frac{2}{3} \frac{\mu^{2}}{m^{2}} \frac{<0\left|\mathbf{p}^{2} / 2 m\right| 0>^{2}}{\tilde{\epsilon}_{1}-\epsilon_{0}}
$$

This is valid for $\bar{\epsilon}_{1}-\epsilon_{0}>0$ which is a severe limitation. In the harmonic case, for example, one has $\tilde{\epsilon}_{1}-\epsilon_{0}=5 w / 2-3 v / 2$ and thus one obtains $\Omega \leq 3\left(v^{2}-w^{2}\right)^{2} /\left(4 v^{2}(5 w-3 v)\right)$ valid for $v<5 w / 3$, i.e. $\alpha<4.4$ in the polaron case. Although this may be improved by better bounds and trial wave functions the appearance of the first excited state energy remains a major disadvantage of this approach.

Instead it seems to be better to deal directly with the path integral (16). Using the concavity of the logarithmic function (or simply looking at a graph of $\log x$ ) the following inequality can be easily derived

$$
\log B \leq \log B_{\text {trial }}+\frac{B}{B_{\text {trial }}}-1
$$

which is valid for $B, B_{\text {trial }}>0$ [26]. Several choices are possible for the trial functional $B_{\text {trial }}$ : one may choose a quadratic form in $\mathbf{x}(t)$ which would be exact for harmonic interactions ( cf. ref. [27] ) but has the disadvantage of being inadequate for strong coupling. Instead let us take the translationally invariant trial functional

$$
B_{\text {trial }}[\mathbf{R}, \mathbf{x}]=C \exp \left(-\int_{0}^{\beta} d t \tilde{V}(\mathbf{R}-\mathbf{x})\right)
$$

with a constant $C$ and a potential function $\tilde{V}$ to be varied. It is easily seen that the path integrals which occur if eqs. $(33,34)$ are used in eq. (16) can be translated into quantum-mechanical expectation values involving the three-body Hamiltonian

$$
\hat{H}_{3}=\frac{\mathbf{P}^{2}}{2(2 M+m)}+\frac{\mathbf{p}_{1}^{2}}{2 \mu}+V\left(\mathbf{r}_{1}\right)-\tilde{V}\left(\mathbf{r}_{1}\right)+\frac{\mathbf{p}_{2}^{2}}{2 \mu}+V\left(\mathbf{r}_{2}\right)+\frac{\mathbf{p}_{1} \cdot \mathbf{p}_{2}}{m} .
$$

Varying the constant $C$ gives

$$
\Omega[\tilde{V}] \leq 2 \epsilon_{0}^{(2)}-<0|\tilde{V}| 0>-\epsilon_{0}^{(3)}
$$

where $\epsilon_{0}^{(3)}$ is the ground-state energy associated with the Hamiltonian $\hat{H}_{3}$. In deriving eq. (36) use has been made of the relation

$$
\lim _{\beta \rightarrow \infty} \frac{1}{\beta} \int_{0}^{\beta} d t<\tilde{V}(\mathbf{r}(t))>s_{\text {trial }}=<0|\tilde{V}(\mathbf{r})| 0>
$$


between path integral averages and ground state expectation values which, e.g., follows from eq. (11b). Variation with respect to $\tilde{V}$ shows that for strong coupling $\tilde{V} \rightarrow V$. In this case $\epsilon_{0}^{(3)} \rightarrow \epsilon_{0}^{(2)}$ and therefore one obtains $\Omega \leq \epsilon_{0}-<0|V| 0>$ which is the correct strong coupling limit (28) and leads to the Pekar limit for the self-energy. For small coupling, however, first order perturbation theory gives

$$
\epsilon_{0}^{(3)}=2 \epsilon_{0}+<00|U| 00>+\mathcal{O}\left(U^{2}\right)
$$

taking $U \equiv \mathbf{p}_{1} \cdot \mathbf{p}_{2} / m-\tilde{V}$ as perturbation. This has the consequence that $\tilde{V}$ cancels in eq. (36) in first order and since it only acts on one particle one obtains in second order

$$
\Omega \leq \frac{1}{m^{2}} \sum_{a, b} \frac{\left|\mathbf{p}_{0 a} \cdot \mathbf{p}_{0 b}\right|^{2}}{\epsilon_{a}+\epsilon_{b}-2 \epsilon_{0}}+\sum_{n \neq 0} \frac{\left|\tilde{V}_{0 n}\right|^{2}}{\epsilon_{n}-\epsilon_{0}}+\mathcal{O}\left(U^{3}\right)
$$

Obviously the best which can be achieved by varying $\tilde{V}$ is $\tilde{V}=\mathcal{O}\left(\alpha^{2}\right)$ so that it does not contribute to eq. (38) in the order considered. Comparing with eq. (25) one then sees that the present variational bound only gives $\Omega \leq 2 \Omega^{(2)}$ at small coupling constants.

Although this is considerably better than Luttinger and Lu's result it is unclear a priori whether the variational determination of the best potential $V(r)$ compensates for this deficiency at small coupling constants. To answer this question one has to minimize $\Omega+<S_{1}>_{s_{\text {trial }}}$ with respect to the potential $V(r)$ which up to now was considered to be fixed. In the polaron case it is well known that for small coupling the ground state energy has the expansion

$$
E_{0}=-\alpha-c_{2} \alpha-\ldots
$$

and that the exact result is $c_{2}=0.015920$ whereas the Feynman approximation gives $c_{2}^{F}=1 / 81=$ 0.012346. In Appendix B it is shown that in the present approach the best potential is not harmonic at small coupling and that the corresponding coefficient is $c_{2}^{S}=(1-8 /(3 \pi)) / 12=0.012598$. This is the same result as obtained by Adamowski et al. [28] and Saitoh [29] who considered the most general quadratic trial action and represents only a small improvement over Feynman's result. In addition, Appendix $B$ demonstrates that an insufficient kinetic term $\Omega$ such that at small coupling $\Omega \rightarrow \lambda \Omega^{(2)}$ with a constant $\lambda \geq 1$ translates into $c_{2} \rightarrow c_{2}^{S} / \lambda$. Thus the aforementioned factor 2 in the variational three-body bound for $\Omega$ renders the present approach in the polaron case still inferior to Feynman's for $\alpha<1$ although it will do better for larger $\alpha$ since it embodies the correct large-coupling limit.

Finally I would like to mention that in principle one could derive better bounds by sharpening the logarithmic inequality (33) to

$$
\log x \leq \sum_{k=1}^{2 l+1} \frac{(-)^{k+1}}{k}(x-1)^{k}
$$

which is valid for $x>0$. However, this would require solving a quantum-mechanical $(2 l+3)$-body problem and thus does not seem practical beyond $l=0$. Already the 3 -body bound (36) poses a formidable numerical problem for intermediate coupling constants. 


\section{Summary}

I have given an improved description of an approximate mapping of (nonrelativistic) field theory to many-body quantum mechanics following the work of Luttinger and Lu. The physical picture associated with this approximation is simple and appealing: the cloud of bosons surrounding the fermion is represented by a "shadow" particle which interacts with the fermion via an effective potential. Surprisingly enough it is not the interaction term but the kinetic term in the variational principle which is the challenging part for analytic evaluation of the various averages. The variational bound derived for this term in the present work requires in general the solution of a 3-body problem with separable two-body interactions. Compared with the exactly solvable case of a harmonic potential it is still inferior to Feynman's ansatz for small coupling constants but eventually becomes better as it has built in the correct large-coupling limit. This can be traced back to the use of an additional inequality for the logarithmic function. As the motivation of the present study was to avoid the double use of Jensen's inequality which badly affected Luttinger and Lu's result this outcome is not fully satisfactory. However, it should be kept in mind that for small coupling constants perturbation theory is available and it is at intermediate and large coupling constants where nonperturbative methods are badly needed. In these cases the present variational bounds might yield useful results.

The relevance of such an approach is not only that it is a very successful nonperturbative method in the polaron case. As the particle-shadow interaction is formulated in ordinary quantum-mechanics it may also be expected that spin- and isospin-dependent interactions (as in the pion-nucleon case) can be handled similarly. Due to the fact that spin in a path integral inherently involves complex actions $[30,31]$, I expect that the variational solutions for the self-energy in this case are only stationary and no longer upper bounds for the true energy. Finally, I note that such "shadows" have been introduced phenomenologically in models for the confinement of quarks in quantum chromodynamics [32]. The main feature there is a nonhermitean constant effective potential which leads to the eventual decay of free quarks and therefore to their non-observation at asymptotic distances. This may arise from the nonabelian nature of QCD in contrast to the simple Yukawa-type field theories considered here. Needless to say that questions of gauge invariance, relativistic covariance and renormalization which have not been considered here at all, have to be answered before progress into this direction can be made.

\section{Acknowledgements}

I would like to thank Dina Alexandrou and Yang Lu for many discussions and helpful remarks. I am particularly indebted to Yang Lu who pointed out an error in an earlier version of the paper. Critical comments by Milan Locher helped to improve the manuscript. 


\section{References}

[1] H. Fröhlich, Phil. Mag. Suppl. 3 (1954) 325

[2] C. Rodriguez and V. K. Fedyanin, Sov. J. Part. Nucl. 15 (1984) 390

[3] T. K. Mitra, A. Chatterjee and S. Mukhopadhyay, Phys. Rep. 153 (1987) 91

[4] N. N. Bogoliubov Jr. and V. N. Plechko, Riv. Nuovo Cimento 11 (1988) 1

[5] M. Bolsterli, in: Adv. Nucl. Phys., eds. J. W. Negele and E. Vogt, vol. 11 (1979) , p. 367

[6] R. Rosenfelder, in: Dirkfest '92, eds. W. W. Buck, K. M. Maung and B. D. Serot, World Scientific (1993), p. 165

[7] R. P. Feynman, Phys. Rev. 97 (1955) 660

[8] T. D. Schultz, Phys. Rev. 116 (1959) 526

[9] J. M. Luttinger and C.-Y. Lu, Phys. Rev. B 21 (1980) 4251

[10] S. I. Pekar: Untersuchungen zur Elektronentheorie der Kristalle, Akademie-Verlag, Berlin (1954)

[11] S. Miyake, J. Phys. Soc. Japan 38 (1975) 181

[12] C. Alexandrou and R. Rosenfelder, Phys. Rep. 215 (1992) 1

[13] J. W. Negele and H. Orland: Quantum Many-Particle Systems, Frontier in Physics vol. 68, Addison-Wesley (1988)

[14] W. Thirring: A Course in Mathematical Physics, vol. 3 : Quantum Mechanics of Atoms and Molecules, Springer (1981)

[15] H. A. Bethe and S. Salpeter : Quantum Mechanics of One- and Two- Electron Systems, in: Handbuch der Physik, ed. S. Flügge, vol. 35, Springer (1957)

[16] I. Lindgren and J. Morrison: Atomic Many-Body Theory, Springer (1986)

[17] R. Rajaraman, Phys. Rev. 129 (1963) 265

[18] A. L. Fetter and J. D. Walecka: Theoretical Mechanics of Particles and Continua, McGraw-Hill (1980)

[19] G. Baym: Lectures on Quantum Mechanics, Benjamin (1969)

[20] H. Hellmann: Einführung in die Quantenchemie, Deuticke (1937); R. P. Feynman, Phys. Rev. 56 (1939) 340

[21] Y. Lu and R. Rosenfelder, Phys. Rev. B 46 (1992) 5211 
[22] D. J. Thouless: The Quantum Mechanics of Many-Body Systems, Academic Press (1961)

[23] R. Balian and M. Vénéroni, Ann. Phys. (N. Y.) 187 (1988) 29

[24] G. Temple, Proc. Roy. Soc. A 119 (1928) 276

[25] M. Reed and B. Simon: Methods of Mathematical Physics, vol. IV: Analysis of Operators, Academic Press (1978), p.85

[26] A. Wehrl, Rev. Mod. Phys. 50 (1978) 232

[27] R. P. Feynman and H. Kleinert, Phys. Rev. A 34 (1986) 5080

H. Kleinert, Phys. Lett. B 280 (1992) 251

[28] J. Adamowski, B. Gerlach and H. Leske, in: Functional Integration - Theory and Applications, eds. J.-P. Antoine and E. Tirapegui, Plenum (1981), p. 291

[29] M. Saitoh, J. Phys. Soc. Jpn. 49 (1980) 878

[30] E. Fradkin and M. Stone, Phys. Rev. B 38 (1988) 7215

[31] M. Bergeron, Fortschr. Phys. 40 (1992) 119

[32] M. Becker et al. , Phys. Lett. 267 (1991) 261 


\section{Appendix}

\section{A Summation of Ring Diagrams}

In order to sum up the ring diagrams I will diagonalize the $N+1$-body Hamiltonian

$$
H_{N+1}=H_{0}+V
$$

within a space spanned by the orthonormalized states

$$
\left|\Psi_{0}\right\rangle=|0,0 \ldots 0\rangle \text { and }\left|\Psi_{1}\right\rangle=C \frac{Q_{0}}{H_{0}-E^{(0)}} V\left|\Psi_{0}\right\rangle .
$$

Here $H_{0}$ is the one-body part of the Hamiltonian, $E^{(0)}=N \epsilon_{0}$ denotes the energy of the unperturbed Hartree state and $V=\sum_{i<j} \mathbf{p}_{i} \cdot \mathbf{p}_{j} \mid m$ is the Eckart term. $\left|\Psi_{0}\right\rangle$ and $\left|\Psi_{1}\right\rangle$ are zero'th and first order perturbative wavefunctions, respectively, and $Q_{0}=1-\left|\Psi_{0}\right\rangle\left\langle\Psi_{0}\right|$ denotes the projection operator which excludes the unperturbed ground state.

As usual the ground state energy of a two state system is given by

$$
E_{N}=\frac{1}{2}\left(H_{00}+H_{11}\right)-\frac{1}{2} \sqrt{\left(H_{11}-H_{00}\right)^{2}+4\left|H_{01}\right|^{2}} .
$$

For the matrix elements one finds

$$
\begin{aligned}
& H_{00}=E^{(0)} \\
& H_{01}=\frac{E^{(2)}}{\sqrt{E^{(2)^{\prime}}}} \\
& H_{11}=E^{(0)}+\frac{1}{2 E^{(2)^{\prime}}}\left(E^{(2)}+E^{(3)}\right)
\end{aligned}
$$

with

$$
\begin{aligned}
E^{(2)} & =\left\langle\Psi_{0}\left|V \frac{1}{H_{0}-E^{(0)}} V\right| \Psi_{0}\right\rangle=N(N-1) \Omega^{(2)} \\
E^{(2)^{\prime}} & =\left\langle\Psi_{0}\left|V \frac{1}{\left(H_{0}-E^{(0)}\right)^{2}} V\right| \Psi_{0}\right\rangle=\frac{N(N-1)}{2} \frac{\partial}{\partial \epsilon_{0}} \Omega^{(2)} \\
E^{(3)} & =\left\langle\Psi_{0}\left|V \frac{1}{H_{0}-E^{(0)}} V \frac{1}{H_{0}-E^{(0)}} V\right| \Psi_{0}\right\rangle=N(N-1)(N-2) \Omega^{(3)} .
\end{aligned}
$$

Only constant and linear terms in $(N-1)$ are needed for the calculation of $\Omega$. Expanding eq. (A3) in powers of $(N-1)$ one obtains

$$
E_{N}=\epsilon_{0}+(N-1) \epsilon_{0}-(N-1) \frac{\Omega^{(2) 2}}{\Omega^{(2)}-\Omega^{(3)}}+\mathcal{O}\left((N-1)^{2}\right)
$$

from which eq. (27) follows. The same result is obtained by a variational calculation with

$$
\left|\Psi>=Z_{0}\right| \Psi_{0}>+Z_{1} \mid \Psi_{1}>
$$

as trial function which shows that eq. (A10) is an upper bound for the energy. Consequently, $\Omega^{\text {ring }}$ is a lower bound for the true kinetic term. 


\section{B Variational Solution for Small Coupling}

In this section I derive the small coupling limit of the polaron energy in the present approach. This is done by expanding the variational bound (22) for the energy in powers of $\alpha$ including terms up to order $\alpha^{2}$.

We know that $\Omega^{(2)}$ given in eq. (25) is correct up to this order. Disentangling the energy denominator by a Laplace transform it may be written as

$$
\Omega^{(2)}=\frac{3}{2} \int_{0}^{\infty} d t F^{2}(t)
$$

where

$$
F(t)=\sum_{n} e^{-t\left(\epsilon_{n}-\epsilon_{0}\right)}|<0| p_{z}|n>|^{2}=<0\left|p_{z} e^{-t\left(h-\epsilon_{0}\right)} p_{z}\right| 0>
$$

is the Laplace transform of the single-particle response function associated with the excitation operator $p_{z}$. Rotational symmetry of the ground state has been used as well as the usual polaron units $(\omega=m=1)$.

To obtain the small-coupling limit of the interaction term $\left\langle S_{1}\right\rangle_{S_{\text {trial }}}$ it is convenient to use the Fourier transformed form of eq. (15)

$$
\begin{aligned}
<S_{1}>_{S_{\text {trial }}}=-\frac{\alpha}{\sqrt{2} \mu} \sum_{n} \frac{1}{1+\Delta_{n}} \quad \int d^{3} r d^{3} r^{\prime} \frac{\phi_{0}(\mathbf{r}) \phi_{0}\left(\mathbf{r}^{\prime}\right) \phi_{n}(\mathbf{r}) \phi_{n}\left(\mathbf{r}^{\prime}\right)}{\left|\mathbf{r}-\mathbf{r}^{\prime}\right|} \\
\cdot\left[1-\exp \left(-2 C \sqrt{\left.\left.1+\Delta_{n}\left|\mathbf{r}-\mathbf{r}^{\prime}\right|\right)\right]}\right.\right.
\end{aligned}
$$

where $C=\sqrt{M \mu / 2}$ and $\Delta_{n}=\epsilon_{n}-\epsilon_{0}$ (see eq. (10) of LL). At small coupling $M, \mu=\mathcal{O}(\alpha)$ and therefore one can expand the exponential in eq. (B.3). However, it must be realized that in this limit the radial extension of the polaron is $\mathcal{O}\left(\alpha^{-1 / 2}\right)$ so that one has to expand up to third order. Using orthogonality and completeness of the wavefunctions $\phi_{n}(r)$ one then obtains

$$
<S_{1}>_{S_{\text {trial }}}=-\alpha-\frac{1}{2} \alpha M+\frac{2}{3} \alpha M^{2} \gamma+\mathcal{O}\left(\alpha^{3}\right)
$$

with

$$
\gamma=\sum_{n} \sqrt{1+\Delta_{n}}|<0| \mathbf{r}|n>|^{2}-<0\left|\mathbf{r}^{2}\right| 0>=<0\left|\mathbf{r} \sqrt{1+h-\epsilon_{0}} \mathbf{r}-\mathbf{r}^{2}\right| 0>\geq 0 .
$$

The variational energy is a function of the mass $M$ of the shadow particle and a functional of the potential $V(r)$ between electron and shadow. However, the mass parameter also enters into the wavefunctions $\phi_{n}$ and the energies $\epsilon_{n}$. It is convenient to separate this dependence by scaling

$$
\xi=\sqrt{M} \mathbf{r}
$$

and defining

$$
\bar{V}(\xi)=\lim _{\alpha \rightarrow 0} V\left(\frac{r}{\sqrt{M}}\right) \text { and } \bar{\epsilon}_{n}=\lim _{\alpha \rightarrow 0} \epsilon_{n} .
$$

Then wavefunctions and energies are independent of $M$

$$
\bar{h} \bar{\phi}_{n}(\xi) \equiv\left(-\frac{1}{2} \Delta_{\xi}+\bar{V}(\xi)\right) \bar{\phi}_{n}(\xi)=\bar{\epsilon}_{n} \phi_{n}(\xi) .
$$


Similar one defines

$$
\bar{\Omega}=\lim _{\alpha \rightarrow 0} \frac{\Omega^{(2)}}{M^{2}} \text { and } \bar{\gamma}=\frac{4}{3} \lim _{\alpha \rightarrow 0} M \gamma
$$

with $M$-independent (i.e. $\alpha$-independent ) quantities $\bar{\Omega}$ and $\bar{\gamma}$. The variational bound for the ground state energy now reads

$$
E_{0}[M, V] \leq M^{2} \bar{\Omega}-\alpha-\frac{1}{2} \alpha M+\frac{1}{2} \alpha M \bar{\gamma}+\mathcal{O}\left(\alpha^{3}\right)
$$

and the variation with respect to $M$ can be performed easily. The optimal mass parameter is

$$
M=\frac{\alpha}{4 \bar{\Omega}}(1-\bar{\gamma})+\mathcal{O}\left(\alpha^{2}\right)
$$

and the energy has the desired expansion

$$
E_{0}=-\alpha-c_{2} \alpha^{2}+\mathcal{O}\left(\alpha^{3}\right)
$$

with

$$
c_{2}=\frac{(1-\bar{\gamma})^{2}}{16 \bar{\Omega}}
$$

In order that the mass of the shadow particle is positive one obviously needs $\bar{\gamma} \leq 1$. Note that the coefficient $c_{2}$ depends inversely on $\bar{\Omega}:$ if the small coupling expansion would have been not performed with $\Omega^{(2)}$ but with $\lambda \Omega^{(2)}$ where $\lambda \geq 1$, the coefficient $c_{2}$ would have been reduced by a factor $1 / \lambda$.

The task is now to maximise $c_{2}$ with respect to the potential $\bar{V}(\xi)$. At first sight this seems impossible to do analytically due to the complicated nonlinear dependence of wavefunctions and energies on $\bar{V}(\xi)$. Still it is possible if not the potential but the resulting response function $\bar{F}(t)$ is varied instead. This requires that the kinetic term $\bar{\Omega}$ can also be expressed in terms of $\bar{F}(t)$. From eqs. (B.5) and (B.9) one first derives

$$
\bar{\gamma}=\frac{2}{\sqrt{\pi}} \int_{0}^{\infty} d t \frac{e^{-t}}{t^{3 / 2}}[\bar{G}(0)-\bar{G}(t)]
$$

where

$$
\bar{G}(t)=\sum_{n} e^{-t\left(\epsilon_{n}-\epsilon_{0}\right)}|<0| \xi_{z}|n>|^{2}=<0\left|\xi_{z} e^{-t\left(h-\bar{\epsilon}_{0}\right)} \xi_{z}\right| 0>
$$

is the Laplace transform of the response function associated with the (scaled) dipole operator $\xi_{z}$. By means of the Heisenberg equations for the dipole operator one immediately finds $\bar{F}(t)=\bar{G}^{\prime \prime}(t)$ and after suitable integrations by parts one obtains the desired relation

$$
\bar{\gamma}=1-4 \int_{0}^{\infty} d t\left[\left(t+\frac{1}{2}\right) \operatorname{erfc}(\sqrt{t})-\sqrt{\frac{t}{\pi}} e^{-t}\right] \bar{F}(t) \equiv 1-\int_{0}^{\infty} d t f(t) \bar{F}(t)
$$

Here $\operatorname{erfc}(x)$ is the complementary error function. Before performing the variation with respect to the function $\vec{F}(t)$ one should realize that it is not completely arbitrary even for a general local potential : expanding $\bar{G}(t)$ in powers of $t$ one finds

$$
\bar{G}(t)=<0\left|\xi_{z}^{2}\right| 0>-t<0\left|\xi_{z}(\bar{h}-\bar{\epsilon}) \xi_{z}\right| 0>+\ldots=<0\left|\xi_{z}^{2}\right| 0>-\frac{t}{2}<0\left|\left[\xi_{z},\left[\bar{h}, \xi_{z}\right]\right]\right| 0>+\ldots
$$


The linear term is identical with the Thomas-Reiche-Kuhn sum rule to which a local potential does not contribute. Evaluating the double commutator using eq. (B.8) one therefore finds

$$
-\bar{G}^{\prime}(0)=\int_{0}^{\infty} d t \bar{F}(t)=\frac{1}{2}
$$

as constraint. The variation of $c_{2}$ can now be performed: with the ansatz

$$
\bar{F}(t)=\frac{w}{2} e^{-w t}
$$

one obtains $w^{\max }=3$ and $c_{2}^{\max }=1 / 81$ which is the small-coupling limit of Feynman's approximation. However, one can do better by varying with respect to the full function $\bar{F}(t)$ under the constraint (B.18). A simple calculation yields

$$
\bar{F}(t)^{\max }=\frac{f(t)}{2 \int_{0}^{\infty} d t f(t)}
$$

and

$$
c_{2}^{\max }=\frac{1}{24} \int_{0}^{\infty} d t f^{2}(t)
$$

with $f(t)$ being defined in eq. (B.16). The integrals involving the error function can all be performed analytically by appropriate integrations by parts and one obtains

$$
c_{2}^{\max }=\frac{1}{12}\left(1-\frac{8}{3 \pi}\right)
$$

Using eq. (B.11) the corresponding mass of the shadow particle is $M=\alpha / 6+\mathcal{O}\left(\alpha^{2}\right)$ which agrees with the small-coupling expansion of the polaron effective mass $m^{\star} \simeq 1+M$. It is unclear which local potential generates generates $\bar{F}(t)^{\max }$ in eq. (B.20) but it certainly is not harmonic as the comparison with eq. (B.19) shows. 


\section{Figure captions}

Fig. 1 :

Second-order Goldstone diagram for the kinetic term $\Omega$ with the Eckart term as two-body interaction. The rules are the same as for the evaluation of the ground-state energy [16] except for an additional factor $-\left(n_{\ell}-1\right)$ where $n_{\ell}$ is the number of loops.

Fig. 2 :

Third-order Goldstone diagrams for $\Omega$.

Fig. 3 :

Schematic drawing of the particle-shadow system together with the shadow replicas as described by the Hamiltonian (19). The dashed lines represent the potential $V\left(\mathbf{x}-\mathbf{R}_{\boldsymbol{i}}\right)$ between the particle and the shadows, whereas the size of the circles indicates the corresponding masses : (a) small coupling constants where $m \gg M$, (b) large coupling constants for which $m \ll M$. 


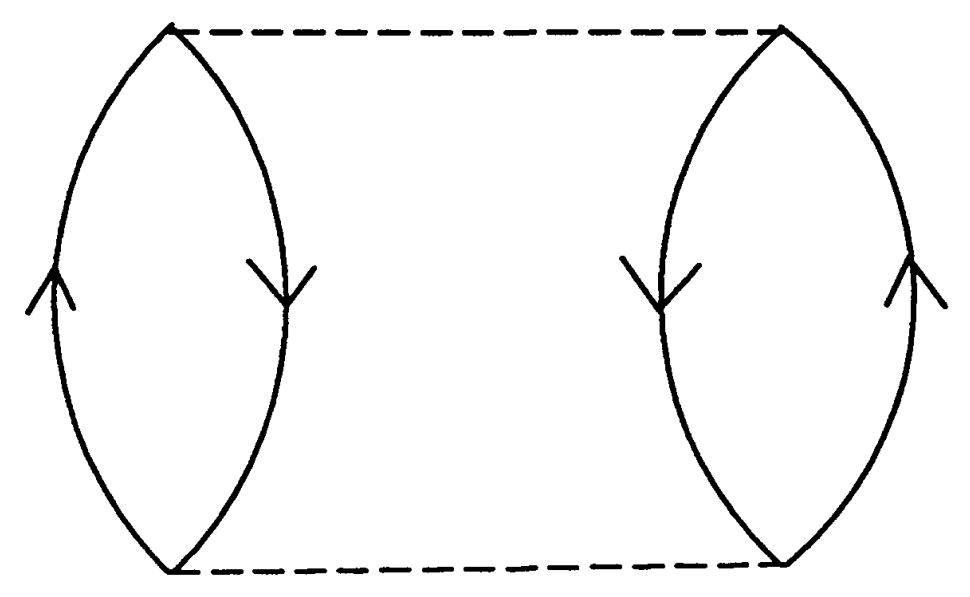

F i g . 1 


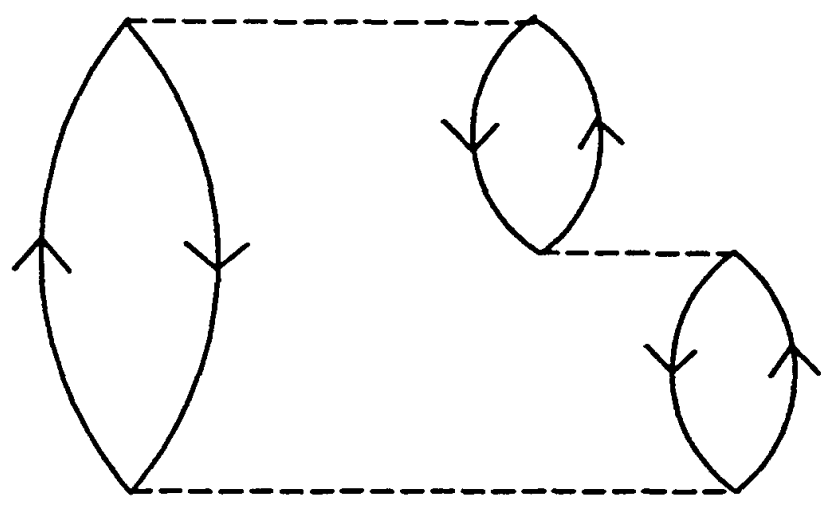

Fig. 2 a

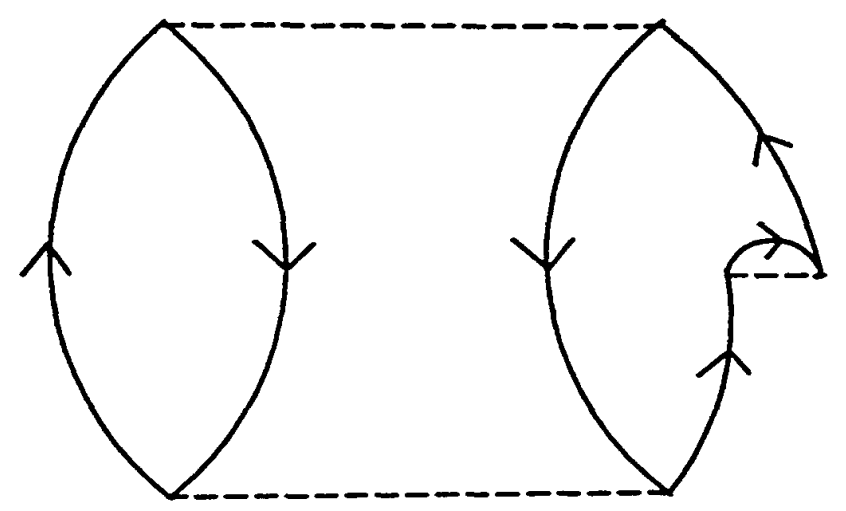

F i g $\quad 2$ c

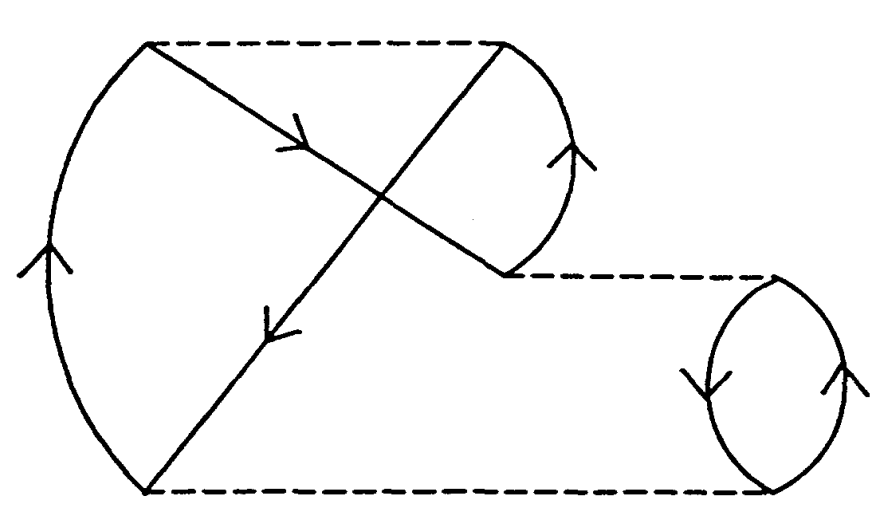

Fi g $\quad 2$ b

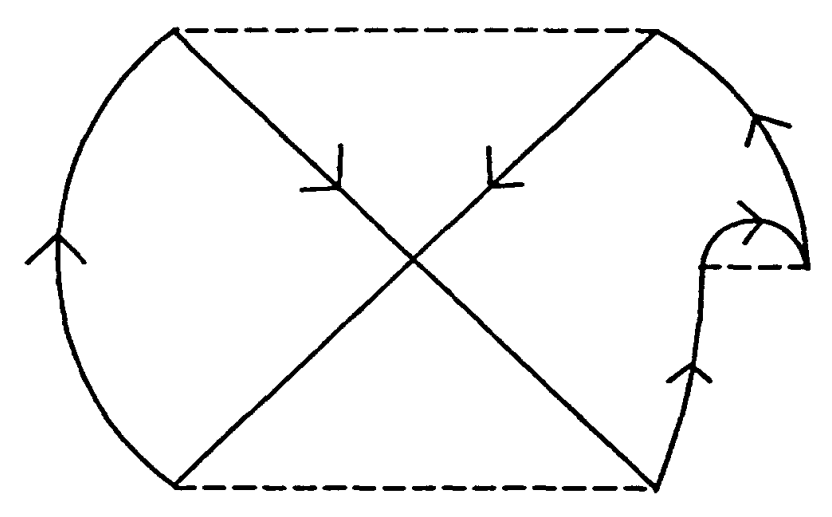

F i g $\quad 2$ d 


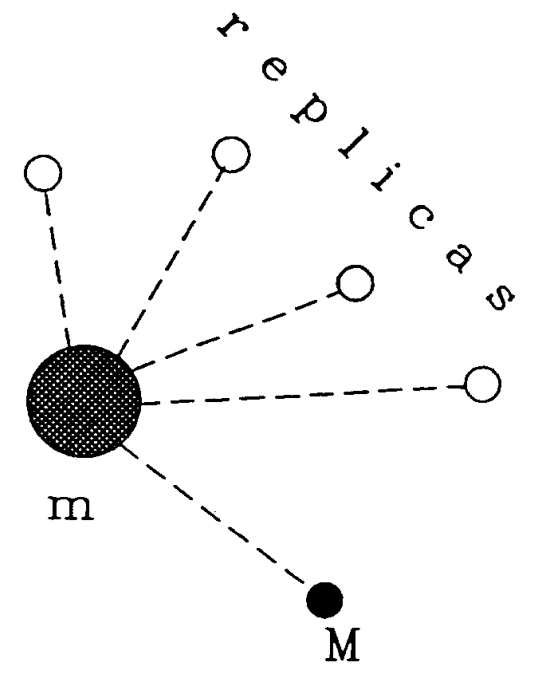

F i g. 3 a

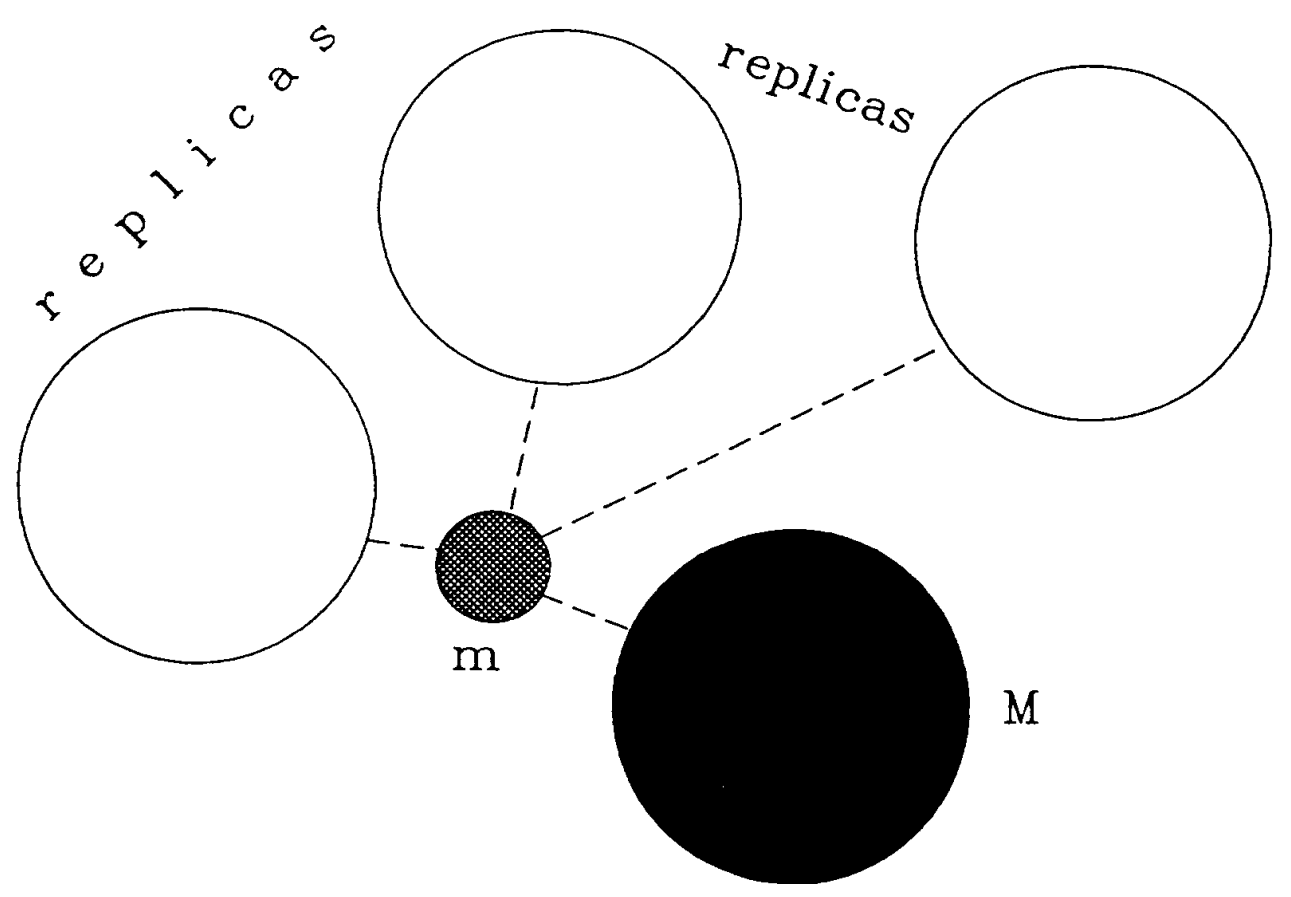

F i g. $3 \mathrm{~b}$ 
